\title{
Magnetic Resonance Imaging of the Neck with and without Contrast
}

National Cancer Institute

\section{Source}

National Cancer Institute. Magnetic Resonance Imaging of the Neck with and without

Contrast. NCI Thesaurus. Code C137920.

Magnetic resonance imaging of the neck with and without a contrast agent to enhance the image. 